\begin{tabular}{|c|l|}
\hline Title & Life span of smooth solutions to the complex Ginzburg-Landau ty pe equation on torus \\
\hline Author(s) & Ozawa, T.; Y amazaki, Y. \\
\hline Citation & Hokkaido University Preprint Series in Mathematics, 599, 1-8 \\
\hline Issue Date & 2003-09 \\
\hline DOI & 10.14943/83744 \\
\hline Doc URL & http://hdl.handle.net/2115/69348 \\
\hline Type & bulletin (article) \\
\hline File Information & pre599.pdf \\
\hline
\end{tabular}

Instructions for use 
Life-Span of Smooth Solutions to the Complex Ginzburg-Landau Type Equation on Torus

T. Ozawa and Y. Yamazaki

Series \#599. September, 2003 


\section{HOKKAIDO UNIVERSITY PREPRINT SERIES IN MATHEMATICS}

\#573 S. Izumiya, D. Pei and M. C. Romero-Fuster, The horospherical geometry of surfaces in Hyperbolic 4-space, 17 pages. 2002.

\#574 S. Izumiya and M. C. Romero-Fuster, The hyperbolic Gauss-Bonnet type theorem, 10 pages. 2002.

\#575 S. Izumiya and S. Janeczko, A symplectic framework for multiplane gravitational lensing, 19 pages. 2002.

\#576 S. Izumiya, M. Kossowski, D. Pei and M. C. Romero-Fuster, Singularities of $C^{\infty}$-lightlike hypersurfaces in Minkowski 4-space, 18 pages. 2002.

\#577 S. Izumiya, D. Pei and M.Takahashi, Evolutes of hypersurfaces in Hyperbolic space, 21 pages. 2002.

\#578 Y. Giga, S. Matsui and S. Sasayama, Blow up rate for semilinear heat equation with subcritical nonlinearity, 29 pages. 2002.

\#579 M. Tsujii, Physical measures for partially hyperbolic surface endomorphisms, 71 pages. 2003.

\#580 Y. Giga and K. Yamada, On viscous Burgers-like equations with linearly growing initial data, 19 pages. 2003.

\#581 T. Nakazi and T. Osawa, Spectra of Toeplitz Operators and Uniform Algebras, 9 pages. 2003.

\#582 Y. Daido, M. Ikehata and G. Nakamura, Reconstruction of Inclusions for the Inverse Boundary Value Problem with Mixed Type Boundary Condition, 18 pages. 2003.

\#583 Y. Daido and G. Nakamura, Reconstruction of Inclusions for the Inverse Boundary Value Problem with Mixed Type Boundary Condition and Source Term, 26 pages. 2003.

\#584 M.-H. Giga and Y. Giga, A PDE approach for motion of phase-boundaries by a singular interfacial energy, 19 pages. 2003.

\#585 A.A. Davydov, G. Ishikawa, S. Izumiya and W.-Z. Sun, Generic singularities of implicit systems of first order differential equations on the plane, 28 pages. 2003.

\#586 K. Yamauchi, On an underlying structure for the consistency of viscosity solutions, 12 pages. 2003.

\#587 T. Miyao, Momentum operators with a winding gauge potential, 15 pages. 2003.

\#588 Y. Giga and R. Kobayashi, On constrained equations with singular diffusivity, 35 pages. 2003.

\#589 O. Sawada, On analyticity rate estimates of the solutions to the Navier-Stokes equations in Besselpotential spaces, 13 pages. 2003.

\#590 T. Nakazi, Exposed points and extremal problems in $H^{1}$ on a bidisc, 11 pages. 2003.

\#591 Y. Tsai and Y. Giga, A numerical study of anisotropic crystal growth with bunching under very singular vertical diffusion, 9 pages. 2003.

\#592 K. Izuchi, T. Nakazi and M. Seto, Backward shift invariant subspaces in the bidisc II, 17 pages. 2003.

\#593 S. Jimbo, Singular perturbation of domains and Semilinear elliptic equations III, 26 pages. 2003.

\#594 Y. Giga, S. Matsui and S. Sasayama, On blow up rate for sign-changing solutions in a convex domain, 12 pages. 2003.

\#595 T. Nakazi and T. Osawa, Invariant subspaces of finite codimension and uniform algebras, 6 pages. 2003.

\#596 T. Mikami, Monge's problem with a quadratic cost by the zero-noise limit $h$-pass processes, 20 pages. 2003.

\#597 Y. Tonegawa, On stable critical points for a singular perturbation problem, 13 pages. 2003.

\#598 Y. Kashima, A subdifferential formulation of fourth order singular diffusion equations, 26 pages. 2003. 


\title{
LIFE-SPAN OF SMOOTH SOLUTIONS TO THE COMPLEX GINZBURG-LANDAU TYPE EQUATION ON TORUS
}

\author{
T.OZAWA AND Y.YAMAZAKI* \\ Department of Mathematics \\ Hokkaido University \\ Sapporo 060-0810, Japan
}

\begin{abstract}
.
An upper bound of the life-span of smooth solutions to the complex GinzburgLandau equation with periodic boundary conditon in one space dimension is given explicitly in terms of an integral mean of the Cauchy data in the case where the interaction is focusing.
\end{abstract}

Mathematics Subject Classification : 35Q55

*) JSPS Fellow 


\section{§. INTRODUCTION}

We consider the life-span of smooth solutions to the Cauchy problem for the complex Ginzburg-Landau type equation (CGL) of the form

$$
\partial_{t} u-(\lambda+i \alpha) \partial_{x}^{2} u=F(u)+\gamma u
$$

with Cauchy data $u(0, x)=u_{0}(x)$, where $u$ is a complex-valued function of $(t, x) \in$ $\mathbb{R}_{+} \times \mathbb{T}, \partial_{t}=\partial / \partial t, \partial_{x}=\partial / \partial x, \lambda \geq 0, \alpha, \gamma \in \mathbb{R}, F(u)$ represents a nonlinear interaction, and the one dimensional torus $\mathbb{T}$ is realized as the interval $[-\pi, \pi]$ by identifying $-\pi$ and $\pi$. A typical form of $F(u)$ is given by a single power. In this paper we are mainly interested in the nonlinear interaction of the form

$$
F(u)=(\kappa+i \beta)|u|^{p}
$$

with $\kappa, \beta \in \mathbb{R}$, and $p>1$. This differs from the usual one that satisfies the gauge condition: $F(u)=(\kappa+i \beta)|u|^{p-1} u$.

Regarding physical background of (CGL), we refer to [11] and references therein for the latest developments. As for the linear part on $u$, the operator $\partial_{t}-\lambda \partial_{x}^{2}$ has an effect of dissipation if $\lambda>0$, the operator $\partial_{t}-i \alpha \partial_{x}^{2}$ has an effect of dispersion if $\alpha \neq 0$, and the multiplication operator by $\gamma$ work as a damping if $\gamma<0$.

There is a large literature on the study of the Cauchy problem for (CGL) (see for instance $[1,7,8,12,14,15]$ and references therein and $[2-6]$ in the special case $\lambda=0$ with periodic boundary condition). To our knowledge, however, little is known for the blow-up solutions of (CGL) on torus except [13] in the case where $\alpha>\lambda=\gamma=0$ and $F(u)=i|u|^{4} u$. When a dissipation effect disappears with $\lambda=0$, the last nonlinearity works on instability of equipartition between the kinetic and potential parts of the energy through its "focusing" sign and on stability of the total mass through its gauge invariance, both of which are essential in the argument of [13].

The purpose in this paper is to examine how another mechanism comes into play on blow-up of solutions on torus even when $\lambda \neq 0$. To be more specific, we show that a monotonicity property has an effect on the blow-up argument when we single out the real or imaginary part of functionals of solutions. In this paper we are mainly interested in the case where the equation involves the effect of dispersion since much is already known for the dissipative case without dispersion.

The main result in this paper is the following

Theorem 1. (1) Let $\lambda \geq 0$ and $\beta>0$. Let $u_{0}$ satisfy

$$
\operatorname{Im} \int_{\mathbb{T}} u_{0}(x) d x>0 .
$$

Then the maximal existence time $T^{*}$ of smooth periodic solutions $u$ of (CGL) with $u(0, x)=u_{0}(x)$ and (1.1) is estimated by

$$
T^{*} \leq \frac{1}{(p-1) \gamma} \log \left(1+\frac{\gamma}{\beta} M(0)^{1-p}\right)
$$


if $\gamma \neq 0$ and $(\gamma / \beta) M(0)^{1-p}>-1$, and by

$$
T^{*} \leq \frac{1}{(p-1) \beta} M(0)^{1-p}
$$

if $\gamma=0$, where $M(0)$ is the integral mean of $\operatorname{Im} u_{0}$ given by

$$
M(0)=\frac{1}{2 \pi} \operatorname{Im} \int_{\mathbb{T}} u_{0}(x) d x .
$$

(2) Let $\lambda \geq 0$ and $\kappa>0$. Let $u_{0}$ satisfy

$$
\operatorname{Re} \int_{\mathbb{T}} u_{0}(x) d x>0 .
$$

Then the maximal existence time $T^{*}$ of smooth periodic solutions of (CGL) with $u(0, x)=u_{0}(x)$ and (1.1) is estimated by

$$
T^{*} \leq \frac{1}{(p-1) \gamma} \log \left(1+\frac{\gamma}{\kappa} M(0)^{1-p}\right)
$$

if $\gamma \neq 0$ and $(\gamma / \kappa) M(0)^{1-p}>-1$, and by

$$
T^{*} \leq \frac{1}{(p-1) \kappa} M(0)^{1-p}
$$

if $\gamma=0$, where $M(0)$ is the integral mean of Re $u_{0}$ given by

$$
M(0)=\frac{1}{2 \pi} \operatorname{Re} \int_{\mathbb{T}} u_{0}(x) d x
$$

Remark 1. The local existence of smooth periodic solutions of (GCL) with (1.1), namely, $T^{*}>0$, is assumed in Theorem 1. Though a precise description of local existence of solutions in specific function spaces is outside of the purpose of this paper, we only refer to [1,15] for $\lambda>0$ and to [2,5,6] for $\lambda=0$ in the subcritical and critical cases at the level of $L^{2}(\mathbb{T})$, namely, in the case where $1<p \leq 5$. The corresponding results for $p>5$ follows by the standard regularity arguments such as a linearization of the equation and the Sobolev embedding $H^{1}(\mathbb{T}) \hookrightarrow L^{\infty}(\mathbb{T})$.

Remark 2. Theorem 1 is optimal in the following sense. For Part (1), let $\lambda \geq 0=\kappa$ and let $\beta$ and $\gamma$ satisfy $\beta>0, \gamma \neq 0$, and $\gamma / \beta>-1$. Then

$$
u(t, x)=i e^{\gamma t}\left(1-\frac{\beta}{\gamma}\left(e^{(p-1) \gamma t}-1\right)\right)^{-1 /(p-1)}
$$


satisfies (CGL) on the time interval $\left[0, T^{*}\right)$ with $T^{*}=\frac{1}{(p-1) \gamma} \log \left(1+\frac{\gamma}{\beta}\right)$ and $u(0, x)$ $=i$ and therefore $M(0)=1$ and $\operatorname{Im} u(t, x)$ and $\operatorname{Im} \int_{\mathbb{T}} u(t, x) d x$ blow up as $t \uparrow T^{*}$.

Let $\lambda \geq 0, \beta>0$, and $\kappa=\gamma=0$. Then

$$
u(t, x)=i(1-(p-1) \beta t)^{-1 /(p-1)}
$$

satisfies (CGL) on the time interval $\left[0, T^{*}\right)$ with $T^{*}=1 /((p-1) \beta)$ and $u(0, x)=i$ and therefore $M(0)=1$ and $\operatorname{Im} u(t, x)$ and $\operatorname{Im} \int_{\mathbb{T}} u(t, x) d x$ blow up as $t \uparrow T^{*}$.

For Part (2), let $\lambda \geq 0=\beta$ and let $\kappa$ and $\gamma$ satisfy $\kappa>0, \gamma \neq 0$, and $\gamma / \kappa>-1$. Then

$$
u(t, x)=e^{\gamma t}\left(1-\frac{\kappa}{\gamma}\left(e^{(p-1) \gamma t}-1\right)\right)^{-1 /(p-1)}
$$

satisfies (CGL) on the time interval $\left[0, T^{*}\right)$ with $T^{*}=\frac{1}{(p-1) \gamma} \log \left(1+\frac{\gamma}{\kappa}\right)$ and $u(0, x)=$ 1 and therefore $M(0)=1$ and $u(t, x)$ and $\int_{\mathbb{T}} u(t, x) d x$ blow up as $t \uparrow T^{*}$.

Let $\lambda \geq 0, \kappa>0$, and $\beta=\gamma=0$. Then

$$
u(t, x)=(1-(p-1) \kappa t)^{-1 /(p-1)}
$$

satisfies (CGL) on the time interval $\left[0, T^{*}\right)$ with $T^{*}=1 /((p-1) \kappa)$ and $u(0, x)=1$ and therefore $M(0)=1$ and $u(t, x)$ and $\int_{\mathbb{T}} u(t, x) d x$ blow up as $t \uparrow T^{*}$.

Remark 3. The blow-up problem for the nonlinear Schrödinger equations with zero Dirichlet boundary condition is studied in [16,17]. Part of the proof in [16,17] seems applicable to the periodic solutions, though the corresponding assumptions of the Cauchy data are different from those in Theorem 1 since the assumptions in [16,17] are described in terms of the Fourier mode of the real or imaginary part of the Cauchy data with respect to the first positive eigenvalue.

We prove Theorem 1 in Section 2. The proof depends on a simple monotonicity argument. In Section 3, we extend the method of proof to the case where the nonlinearity $F(u)$ has a derivative coupling.

\section{§2. Proof of Theorem 1}

Proof of Part (1). For any $t$ with $0<t<T^{*}$ we define

$$
M(t)=\frac{e^{-\gamma t}}{2 \pi} \operatorname{Im} \int_{\mathbb{T}} u(t, x) d x .
$$


Differentiating $M(t)$, we have

$$
\begin{aligned}
& M^{\prime}(t)=\frac{e^{-\gamma t}}{2 \pi} \operatorname{Im} \int_{\mathbb{T}}\left(-\gamma u+\partial_{t} u\right) \\
& =\frac{e^{-\gamma t}}{2 \pi} \operatorname{Im} \int_{\mathbb{T}}\left((\lambda+i \alpha) \partial_{x}^{2} u+F(u)\right) \\
& =\frac{\beta e^{-\gamma t}}{2 \pi} \int_{\mathbb{T}}|u|^{p} \geq 0 .
\end{aligned}
$$

This implies that $M(t)>0$ for any $t$ with $0 \leq t<T^{*}$ since $M(0)>0$ by assumption. By the Hölder inequality and (2.1), we have

$$
M(t)^{p} \leq \frac{e^{-p \gamma t}}{2 \pi} \int_{\mathbb{T}}|u|^{p}=\frac{e^{-(p-1) \gamma t}}{\beta} M^{\prime}(t),
$$

from which we obtain

$$
\begin{aligned}
& \frac{d}{d t}\left(M(t)^{1-p}\right)=-(p-1) M(t)^{-p} M^{\prime}(t) \\
& \leq-(p-1) \beta e^{(p-1) \gamma t} .
\end{aligned}
$$

Integrating both sides of (2.2), we obtain

$$
M(t)^{1-p} \leq \begin{cases}M(0)^{1-p}-\frac{\beta}{\gamma}\left(e^{(p-1) \gamma t}-1\right) & \text { if } \gamma \neq 0 \\ M(0)^{1-p}-(p-1) \beta t & \text { if } \gamma=0\end{cases}
$$

for any $t$ with $0 \leq t<T^{*}$. Since the LHS of (2.3) is positive for any $t$ with $0 \leq t<T^{*}$, the maximal existence time $T^{*}$ satisfies the inequality

$$
\begin{array}{lll}
\frac{\beta}{\gamma}\left(e^{(p-1) \gamma T^{*}}-1\right) \leq M(0)^{1-p} & \text { if } & \gamma \neq 0 \\
(p-1) \beta T^{*} \leq M(0)^{1-p} & \text { if } & \gamma=0 .
\end{array}
$$

This proves Part (1).

Proof of Part (2). If we define

$$
M(t)=\frac{e^{-\gamma t}}{2 \pi} \operatorname{Re} \int_{\mathbb{T}} u(t, x) d x,
$$

then

$$
M^{\prime}(t)=\frac{\kappa e^{-\gamma t}}{2 \pi} \int_{\mathbb{T}}|u|^{p} \geq 0 .
$$

Part (2) follows by a similar argument as in Part (1). 


\section{§3. An EXtension to DERIVAtive COUPLing}

In this section we show that a similar method works on (CGL) with nonlinearity of derivative type. We consider the nonlinearities of the form

$$
F(u)=(\kappa+i \beta)|u|^{2} \partial_{x} u
$$

Theorem 2. Let $\lambda=0$ and $\beta>0$. Let $u_{0}$ satisfy

$$
\operatorname{Im} \int_{\mathbb{T}} u_{0}(x) \overline{\partial_{x} u_{0}(x)} d x>0
$$

Then the maximal existence time $T^{*}$ of smooth periodic solutions $u$ of (CGL) with $u(0, x)=u_{0}(x)$ and (3.1) is estimated by

$$
T^{*} \leq \frac{1}{2 \gamma} \log \left(1+\frac{\gamma}{\beta M(0)}\right)
$$

if $\gamma \neq 0$ and $\gamma /(\beta M(0))>-1$, and by

$$
T^{*} \leq 1 /(2 \beta M(0))
$$

if $\gamma=0$, where

$$
M(0)=\frac{1}{2 \pi} \operatorname{Im} \int_{\mathbb{T}} u_{0}(x) \overline{\partial_{x} u_{0}(x)} d x
$$

Remark 4. The local existence of smooth periodic solutions of (CGL) with (3.1), namely, $T^{*}>0$, is assumed in Theorem 2 as stated in Remark 1. Regarding local existence results for (CGL) with derivative coupling, we refer to [10], where a general information of applicable gauge transformation technique is presented (see also [9] and references therein).

Remark 5. Theorem 2 is optimal in the following sense. Let $\beta>0$ and $\lambda=\kappa=$ $\gamma=0$. Then

$$
u(t, x)=e^{-i \alpha t-i x}(1-2 \beta t)^{-1 / 2}
$$

satisfies (CGL) with periodic boundary condition on the time interval $\left[0, T^{*}\right)$ with $T^{*}=1 /(2 \beta)$ and $u(0, x)=e^{-i x}$. Therefore $M(0)=1$ and $|u(t, x)|$ and $\operatorname{Im} \int_{\mathbb{T}} u(t, x) \overline{\partial_{x} u(t, x)} d x$ blow up as $t \uparrow T^{*}$. 
Remark 6. The only functional in the argument below corresponds to the linear momentum. In the standard blow-up argument on the nonlinear Schrödinger equations, the most important functional corresponds to the variance or the moment of inertia, while the linear momentum plays an auxiliary role.

Proof of Theorem 2. For any $t$ with $0<t<T^{*}$ we define

$$
M(t)=\frac{e^{-2 \gamma t}}{2 \pi} \operatorname{Im} \int_{\mathbb{T}} u(t, x) \overline{\partial_{x} u(t, x)} d x .
$$

Differentiating $M(t)$ and integrating by parts, we have

$$
\begin{aligned}
& M^{\prime}(t)=\frac{e^{-2 \gamma t}}{\pi} \operatorname{Im} \int_{\mathbb{T}}\left(\partial_{t} u-\gamma u\right) \overline{\partial u} \\
& =\frac{e^{-2 \gamma t}}{\pi} \beta \int_{\mathbb{T}}|u|^{2}|\partial u|^{2} \geq 0
\end{aligned}
$$

This implies that $M(t)>0$ for any $t$ with $0 \leq t<T^{*}$, since $M(0)>0$ by assumption. By the Schwarz inequality and (3.2), we have

$$
M(t)^{2} \leq \frac{e^{-4 \gamma t}}{2 \pi} \int_{\mathbb{T}}|u|^{2}|\partial u|^{2}=\frac{e^{-2 \gamma t}}{2 \beta} M^{\prime}(t),
$$

from which we obtain

$$
\begin{aligned}
& \frac{d}{d t}\left(M(t)^{-1}\right)=-M(t)^{-2} M^{\prime}(t) \\
& \leq-2 \beta e^{2 \gamma t} .
\end{aligned}
$$

The rest of the proof proceeds in the same way as in the proof of Theorem 1 .

\section{REFERENCES}

[1] T.Abe and N.Okazawa, Global solvability of the complex Ginzburg-Landau equation with distribution-valued initial data, preprint.

[2] J.Bourgain, Fourier transform restriction phenomena for certain lattice subsets and applications to nonlinear evolution equations, Part I: Schrödinger equations, Geom. Funct. Anal. 3 (1993), 107-156.

[3] J.Bourgain, Periodic nonlinear Schrödinger equations and invariant measures, Commun. Math. Phys. 166 (1995), 1-26.

[4] H.Chihara, The initial value problem for Schrödinger equations on the torus, International Math. Res. Notices 2002 (2002), 789-820.

[5] J.Ginibre, Le problème de Cauchy pour des EDP semi-linéaires périodiques en variables d'espace (d'après Bourgain), Séminaire Bourbaki, exposé 796, Astérisque (1995). 
[6] J.Ginibre, An introduction to nonlinear Schrödinger equations, in "Nonlinear Waves" (R.Agemi, Y.Giga, and T.Ozawa, Eds.), GAKUTO International Series, Mathematical Sciences and Applications, Gakkōtosho, Tokyo, 1997.

[7] J.Ginibre and G.Velo, The Cauchy problem in local spaces for the complex Ginzburg-Landau equation I. Compactness methods, Physica D 95 (1996), 191-228.

[8] J.Ginibre and G.Velo, The Cauchy problem in local spaces for the complex Ginzburg-Landau equation II. Contraction methods, Commun. Math. Phys. 187 (1997), 45-79.

[9] N.Hayashi and T.Ozawa, On the derivative nonlinear Schrödinger equation, Physica D 55 (1992), 14-36.

[10] N.Hayashi and T.Ozawa, Remarks on the nonlinear Schrödinger equation in one space dimension, Differential and Integral Equations 7 (1994), 453-461.

[11] C.Hemming and R.Kapral, Front explosion in a resonantly forced complex Ginzburg-Landau system, Physica D 168 (2002), 10-22.

[12] C.D.Levermore and M.Oliver, Distribution-valued initial data for the complex Ginzburg-Landau equation, Commun. PDE 22 (1997), 39-48.

[13] T.Ogawa and Y.Tsutsumi, Blow-up solutions for the nonlinear Schrödinger equation with quartic potential and periodic boundary condition, in "Functional-Analytic Methods for Partial Differential Equations" (H.Fujita, T.Ikebe, and S.T.Kuroda, Eds.), Lecture Notes in Math. 1450 (1989), Springer-Verlag.

[14] N.Okazawa and T.Yokota, Monotonicity method for the complex Ginzburg-Landau equation, including smoothing effect, Nonlinear Analysis TMA 47 (2001), 79-88.

[15] N.Okazawa and T.Yokota, Global existence and smoothing effect for the complex GinzburgLandau equation with p-Laplacian, J.Differential Equations 182 (2002), 541-576.

[16] J.Zhang, On the finite-time behavior of nonlinear Schrödinger eqations, Commun. Math. Phys. 162 (1994), 249-260.

[17] J.Zhang, Finite-time behavior of solutions to nonlinear Schrödinger evolution equations, Nonlinear Analysis, TMA 24 (1995), 165-174. 